\title{
Development of an efficient mutagenesis technique using ion beams: Toward more controlled mutation breeding
}

\author{
Yoshihiro Hase*, Yusuke Akita, Satoshi Kitamura, Issay Narumi, Atsushi Tanaka \\ Quantum Beam Science Directorate, Japan Atomic Energy Agency, Takasaki, Gunma 370-1292, Japan \\ *E-mail: hase.yoshihiro@jaea.go.jp Tel: +81-27-346-9549 Fax: +81-27-346-9688
}

Received December 21, 2011; accepted January 6, 2012 (Edited by Y. Ozeki)

\begin{abstract}
Ion beams have been used as a mutagen to improve the efficiency of plant mutation breeding. Mutation breeding is sometimes perceived as a random process. In this review, we describe our recent progress in developing a more efficient mutagenesis technique using ion beam irradiation combined with sucrose pretreatment or subsequent reirradiation. To shorten the time required for breeding new cultivars of cyclamen, we identified anthocyanin biosynthesis genes and examined the effectiveness of PCR screening of irradiated deletion-mutant candidates at early growth stages. We believe this research is a step toward more efficient and controlled mutation breeding using ion beams.
\end{abstract}

Key words: Anthocyanin, Cyclamen, flower color, ion beam, mutation breeding.

Mutation breeding has provided significant benefits to agriculture. More than 3,000 mutant crop varieties have been produced over the past 60 years (Mutant Variety Database, Joint FAO/IAEA Programme). Most of those varieties were created using ionizing radiation, chiefly gamma-rays. High-energy ion beams that are generated by accelerators have been shown to have greater biological effects than gamma-rays. Increasing evidence suggests that ion beams efficiently induce mutations (Tanaka et al. 2010). Thus, mutation breeding using ion beams is an effective means of generating new plant varieties. However, mutation breeding is sometimes regarded as random, and whether the desired mutant traits can be obtained in the plant material of interest is, in fact, often uncertain. Mutability varies, even between varieties of the same plant species. We carried out this research project to make mutation breeding a more efficient and controlled technique.

We believe that there are three factors important to the success of mutation breeding: the efficiency of mutagenesis, the starting plant material, and mutant screening. The efficiency of mutagenesis is determined by the probability of obtaining the desired mutant traits. The frequency with which desired mutants appear is generally very low. More efficient techniques to induce desired mutations will greatly benefit mutation breeding. The starting plant material is important because the genetic background of the parent plant generally defines the range of mutant traits that can be obtained. Identification of the genes associated with valuable traits would help in understanding the genetic background and choosing suitable starting material. Finally, the efficiency of mutant screening has a great impact on the cost of mutation breeding, especially in species, such as cyclamen, that require long periods before the mutant traits can be evaluated. The ability to screen candidate mutants at an early growth stage based on genetic information associated with the desired traits would be highly advantageous.

This review summarizes our recent work to improve these three important factors in mutation breeding. We describe the development of an efficient mutagenesis technique using ion beam irradiation combined with pretreatment or subsequent re-irradiation. We analyzed flower-color mutants of cyclamen to expand our knowledge about the relationship between mutations of anthocyanin biosynthesis genes and phenotypic traits. We also describe the effectiveness of deletion

Abbreviations: AMT, anthocyanin methyltransferase; ANS, anthocyanidin synthase; Ch2G, chalcone 2'-glucoside; CHI, chalcone isomerase; CHS, chalcone synthase; Cy3,5dG, cyanidin 3,5-diglucoside; DFR, dihydroflavonol 4-reductase; Dp3,5dG, delphinidin 3,5-diglucoside; F3H, flavanone 3-hydroxylase; F3' $\mathrm{H}$, flavonoid 3'-hydroxylase; F3'5' $\mathrm{H}$, flavonoid 3'5'-hydroxylase; FLS, flavonol synthase; GBCP, amphidiploid between C. persicum 'Golden Boy' and C. purpurascens; GST, glutathione S-transferase; 2'GT, chalcone 2'-glucosyltransferase; 3GT, anthocyanidin 3-glucosyltransferase; 5GT, anthocyanin 5-glucosyltransferase; KM, fragrant cyclamen cultivar-'Kaori-no-mai'; LET, linear energy transfer; Mv3G, malvidin 3-glucoside; Mv3,5dG, malvidin 3,5-diglucoside; Pn3Nh, peonidin 3-neohesperidoside; Pn3,5dG, peonidin 3,5-diglucoside; UR, fragrant cyclamen cultivar-'Uruwashi-no-kaori'.

This article can be found at http://www.jspcmb.jp/

Published online June 15, 2012 
mutant screening at early growth stages of mutagenized cyclamen.

\section{Efficient induction of flower color mutants by sucrose pretreatment}

It is a generally accepted idea that ionizing radiation induces mutations randomly throughout the genome. The ability to efficiently induce mutations for a particular phenotypic trait, i.e., to control of the direction of mutations, would be a major breakthrough. However, no effective methods have been established to control the direction of mutations in practical plant breeding. Nagatomi et al. (1997) examined the mutagenic effects of ion beams in chrysanthemum and reported that flower color mutants were obtained with higher frequency when cultured petals rather than cultured leaves were irradiated. Although the underlying mechanism was not clear, they hypothesized that the genes involved in flower color synthesis were relatively mutable where those genes were highly expressed.

We examined whether the condition of the cells altered the mutation frequency of genes affecting particular phenotypes using the violet-flowered petunia (Hase et al. 2010). Sugars are known to stimulate anthocyanin accumulation (Hara et al. 2003; Mita et al. 1997; Tsukaya et al. 1991). We found that the addition of $3 \%(88 \mathrm{mM})$ sucrose greatly stimulated anthocyanin accumulation in petunia seedlings. Two major anthocyanins with absorption peaks near $532 \mathrm{~nm}$ were detected in the seedlings treated with sucrose. These facts suggested that the expression of genes involved in anthocyanin biosynthesis could be activated by sucrose treatment in petunia seedlings. These two anthocyanins were also abundant in mature petals. Treatment with $88 \mathrm{mM}$ sodium chloride had no visible effect on anthocyanin accumulation in seedlings, while $88 \mathrm{mM}$ mannitol or sorbitol were much less effective than sucrose treatment. Six percent sucrose was more effective than $3 \%$, suggesting that the anthocyanin accumulation in petunia seedlings was not an osmotic response (Hase et al. unpublished results). These findings were consistent with Hara et al. (2003), who reported that detached radish hypocotyls treated with sucrose accumulated large amounts of anthocyanins while those treated with mannose or a glucose analog did not accumulate anthocyanins.
To test the hypothesis that mutations are more likely to occur in tissues where the target genes are highly expressed, petunia seedlings with or without sucrose pretreatment were exposed to carbon ions, and the $M_{2}$ seeds were collected from individual $\mathrm{M}_{1}$ plants. Carbon ion irradiation combined with sucrose pretreatment increased the frequency of flower-color mutants 2.6fold compared with carbon ion irradiation alone (Table 1). The frequency of chlorophyll mutants, which was examined as a reference, was not significantly affected by sucrose pretreatment. Our results suggested that the direction of mutation could be controlled to some extent by the ion beam irradiation combined with certain kinds of pretreatment, possibly by changing the profile of gene expression.

\section{Stepwise trait improvement using consecutive ion beam irradiation}

The advantage of mutation breeding lies in its ability to improve one or a few desirable traits without altering the remaining characteristics. Ion beams are better for this purpose than low-linear energy transfer (LET) radiation because energy deposition is highly localized along the path of ion particles. In fact, ion beams induce mutations with lower doses than did low-LET radiation. The mutation frequency in Arabidopsis using carbon ions was 20-fold higher per unit dose than that using electron beams (Shikazono et al. 2005). Therefore, we anticipated that consecutive irradiation with ion beams would improve desirable traits in a stepwise manner. In this research project, we applied stepwise improvement using ion beams to fragrant cyclamen. Three cultivars of fragrant cyclamen were created by crossing cyclamen cultivars (Cyclamen persicum) with the scented cyclamen species C. purpurascens (Ishizaka 2008). One aim of this research project was to create cultivars of fragrant cyclamen with novel petal coloration, because currently only pink and purple flowers are available.

Etiolated petioles prepared from the fragrant cyclamen cultivar 'Uruwashi-no-kaori' (UR; Figure 1B), which has a flower with pink corolla lobes called 'slips' and a deep-purple center called the 'eye', were exposed to carbon ions. The percentage of explants that generated adventitious shoots was greatly decreased at $5 \mathrm{~Gy}$ and above. Twenty-three of 1,079 regenerated plants showed variation in flower color and/or shape. The salmon-

Table 1. Effects of sucrose pretreatment on the frequency of flower-color and chlorophyll mutants.

\begin{tabular}{lcc}
\hline & $\begin{array}{c}\text { Number of } \mathrm{M}_{2} \text { lines containing } \\
\text { flower-color mutants }\end{array}$ & $\begin{array}{c}\text { Number of } \mathrm{M}_{2} \text { lines containing } \\
\text { chlorophyll mutants }\end{array}$ \\
\hline $\begin{array}{c}\text { Carbon ion irradiation with sucrose } \\
\text { pretreatment }\end{array}$ & $42 / 3,309(1.3 \%)^{*}$ & $17 / 3,373(0.5 \%)$ \\
Carbon ion irradiation only & $15 / 2,960(0.5 \%)$ & $13 / 3,218(0.4 \%)$ \\
\hline
\end{tabular}

* Significantly higher than the value with carbon ion irradiation only $(p<0.01)$. Data are the combined results of three independent experiments reported in Hase et al. (2010). 

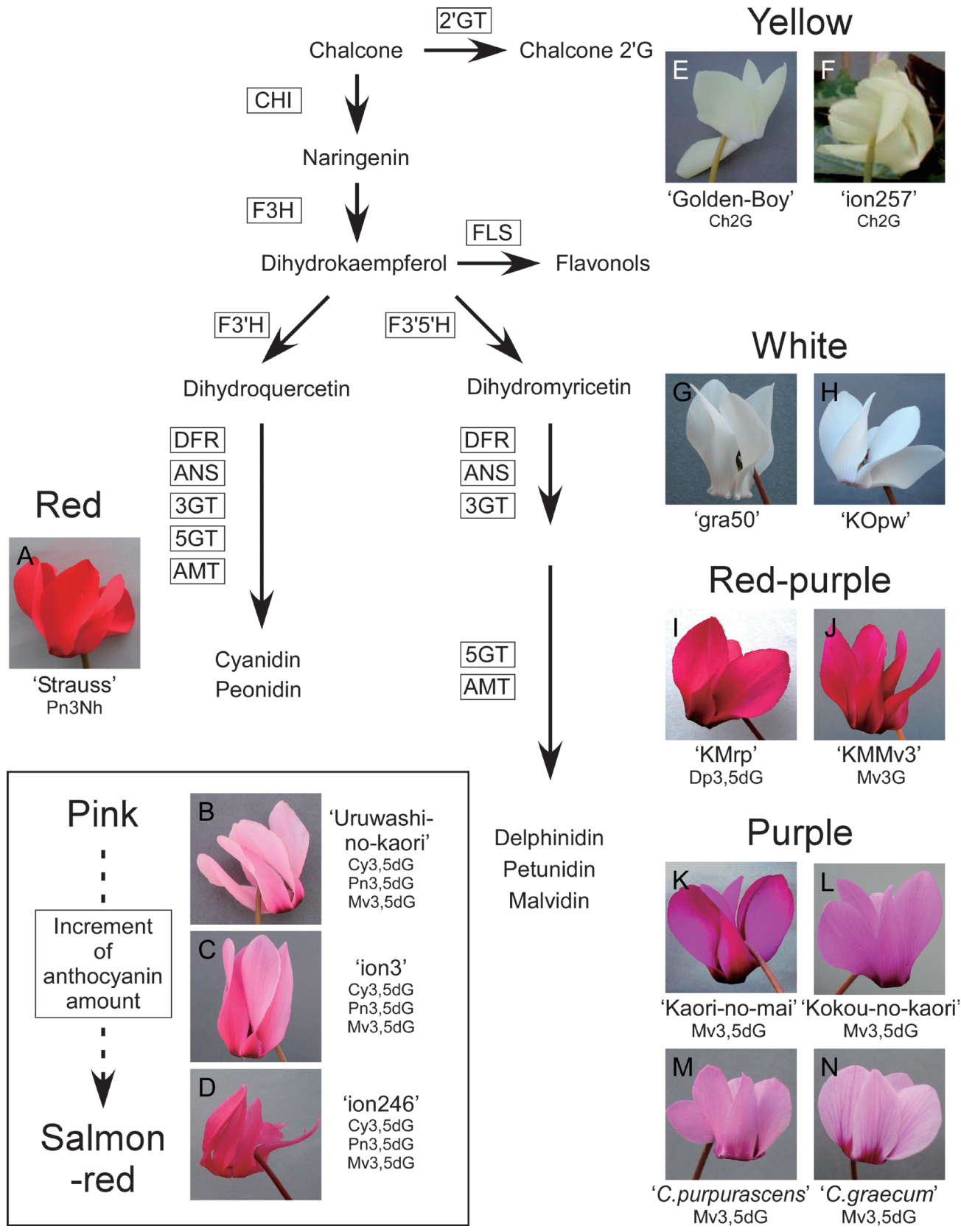

Figure 1. Flowers of cyclamen species, cultivars, and ion-beam induced mutants shown beside the anthocyanin biosynthesis pathway. Flowers are grouped according to coloration. The boxed panel (below left) shows incremental improvement in anthocyanin content induced by consecutive irradiation with ion beams. The strain name and major anthocyanin components are below each flower. Cyclamen persicum cultivars 'Strauss' (A) and 'Golden boy' (E); fragrant cyclamen cultivars (C. persicum $\times$ C. purpurascens) 'Uruwashi-no-kaori', UR (B), 'Kaori-no-mai', KM (K) and 'Kokou-nokaori', KO (L); wild species C. purpurascens (M) and C. graecum (N); salmon-red-flowered mutants, 'ion3' (C) and 'ion246' (D) obtained from UR; a yellow-flowered mutant 'ion257' obtained from 'Golden boy' $\times$ C. purpurascens (GBCP) (F); a white-flowered variant 'gra50' derived from C. graecum (G); a white-flowered mutant 'KOpw' obtained from KO (H); red-purple-flowered mutants, 'KMrp' (I) and 'KMMv3' (J), obtained from KM.

red variant 'ion3' (Figure 1C) was obtained from the population irradiated with $0.1 \mathrm{~Gy}$. In an effort to obtain a red variant, etiolated petioles prepared from ion 3 were exposed again to carbon ions with doses ranging from 0 to $5 \mathrm{~Gy}$. Dose-response for the regeneration rate showed a similar trend as in the first round of carbon-ion irradiation. The deep salmon-red variant, named 'ion246' (Figure 1D), was obtained from the population irradiated with $0.5 \mathrm{~Gy}$. Interestingly, the petals of ion 246 have no eye region, unlike the petals of both ion 3 and UR. Thus, the two rounds of ion beam irradiation were effective to increase the reddish coloration in fragrant cyclamen in a stepwise manner.

In recent years, stepwise improvement with ion beams has been applied to other ornamentals. Two or three rounds of carbon-ion irradiation increased flower 
variations in Osteospermum (Iizuka et al. 2008; Okada et al. 2009). Stepwise improvement with ion beams also succeeded in chrysanthemum; a pure-white variant was obtained from purplish-red chrysanthemums (Sato et al. 2006). Ueno et al. (2006) successfully imparted two agronomic traits, an early-flowering phenotype and fewer lateral buds, to chrysanthemum with two rounds of carbon ion irradiation. In all of these studies, the plant tissues tended to be more sensitive to radiation during the second round of irradiation than the first. Ueno et al. (2006) recommended measuring cellular DNA content to assess radiation sensitivity because mutant plants, which had lower cellular DNA content than their parents, generally showed higher radiation sensitivity. However, in the case of cyclamen, there was no noticeable difference in radiation sensitivity between the first and second round of irradiation. This was probably because ion3 was obtained with a very low dose, although the cellular DNA content was not known. These results suggest that consecutive irradiation with ion beams could be effective to improve desirable traits in a stepwise manner if proper irradiation doses are applied.

\section{Identification of anthocyanin biosynthesis gene homologues in C. purpurascens}

Cyclamen is one of the most popular potted ornamental plants. In mutation breeding, seeds or cultured tissues are often mutagenized. However, cyclamen generally takes about two years to flower and allow flower traits to be evaluated. Screening mutant candidates at earlier growth stages would improve the efficiency of mutation breeding of cyclamen. To do this, we must expand our knowledge about the relationships among gene mutation, anthocyanin components, and flower coloration in cyclamen. In this section, we describe analyses of flower color mutants and identify genes that affect flower coloration in cyclamen.

The genes involved in anthocyanin biosynthesis are highly conserved among species. Sequences of several anthocyanin biosynthesis gene homologues of $C$. persicum cultivars have been deposited in the GenBank database, although little information is available regarding the expression and functions of those genes. The major anthocyanins found in cyclamen cultivars are cyanidin 3,5-diglucoside (Cy3,5dG), peonidin 3,5-diglucoside (Pn3,5dG), and malvidin 3,5-diglucoside (Mv3,5dG) (Takamura et al. 2000; 2005). A yellow-flowered cyclamen cultivar that accumulates chalcone $2^{\prime}$-glucoside $(\mathrm{Ch} 2 \mathrm{G})$ is also known (Miyajima et al. 1991). The anthocyanin biosynthesis pathway in cyclamen is outlined in Figure 1. To understand the genetic background of fragrant cyclamen (C. persicum $\times$ C. purpurascens), we identified the homologues of anthocyanin biosynthesis genes from C. purpurascens. Total RNA was isolated from immature

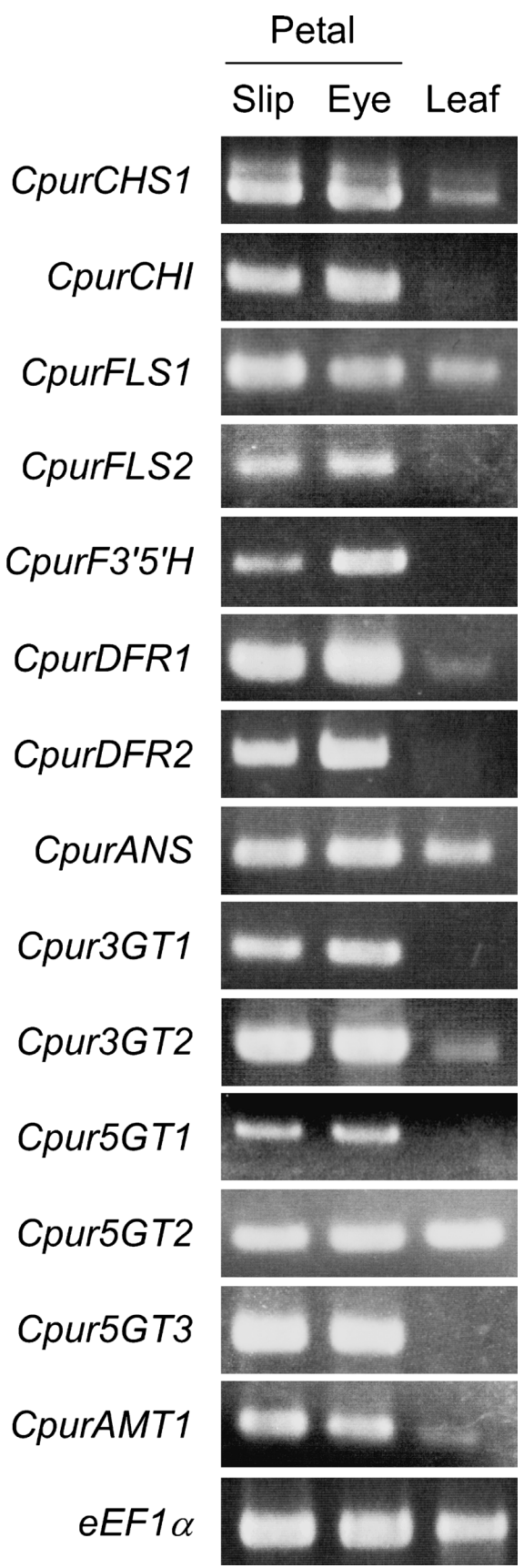

Figure 2. Expression analysis of anthocyanin biosynthesis gene homologues of Cyclamen purpurascens. All images show RT-PCR products after 27 cycles from the 'slip' and 'eye' regions of petals and from leaves. The eEF1 $\alpha$ was used as an internal control.

petals of C. purpurascens, and the cDNA fragments were amplified using degenerate primers designed from known anthocyanin biosynthesis genes. Fourteen fulllength cDNAs were identified, and RT-PCR analysis showed that 11 of them were more highly expressed in petals than leaves (Figure 2; Akita et al. unpublished results). These data suggested that expression of these genes were associated with anthocyanin biosynthesis in petals of C. purpurascens. 
Anthocyanin methyltransferase in C. purpurascens In an effort to produce new flower colors in fragrant cyclamen, the cultivar 'Kaori-no-mai' (KM; Figure $1 \mathrm{~K})$ was mutagenized with ion beams. KM has purple petals that contain Mv3,5dG as a major anthocyanin component. No cyclamen was known that had delphinidin as a major anthocyanin component. We anticipated that we could obtain delphinidin-type cyclamen by inactivating the methylation step from delphinidin to malvidin. A red-purple mutant, 'KMrp' (Figure 1I), was successfully obtained, and the major anthocyanin component was identified as delphinidin 3,5-diglucoside (Dp3,5dG) (Kondo et al. 2009). We further analyzed KMrp at the molecular level to identify the gene responsible for the color differences between KM and KMrp. Please refer to Ishizaka et al. (2012, in this issue) for more details on the generation of the KMrp mutant.

Because the methylation step from delphinidin to malvidin was thought to be disrupted in KMrp, we examined the anthocyanin methyltransferase (AMT) homologues of KM to identify the responsible gene. Four AMT homologues, namely CkmOMT1-4, were identified. While CkmOMT3 and CkmOMT4 were expressed in both petals and leaves, CkmOMT1 and CkmOMT2 were expressed only in petals. In KMrp petals, however, CkmOMT2 expression was not detectable. Genomic PCR failed to amplify any part of the $C k m O M T 2$ coding sequence from KMrp, suggesting that the CkmOMT2 coding region had been deleted. Furthermore, methylation activity was confirmed in vitro using a recombinant CkmOMT2 protein and Dp3,5dG as a substrate. These facts suggest that the failure of CkmOMT2 to be expressed was closely associated with the disruption of methylation step from delphinidin to malvidin in KMrp (Akita et al. 2011).

\section{Dihydroflavonol 4-reductase in C. purpurascens}

Cyclamen graecum is a wild species that has beautiful variegated leaves and resistance to fungi and viruses (Ishizaka 2008). To identify which genes affect flower color in cyclamen, C. graecum (Figure $1 \mathrm{~N}$ ) and its whiteflowered variant 'gra50' (Figure 1G) were comparatively analyzed. C. graecum has pink-purple flowers, and the major anthocyanin component is Mv3,5dG. Anthocyanins were below detectable levels in gra50 petals. The gra50 petals accumulated higher amounts of flavonols than did C. graecum. Those flavonols were kaempferol derivatives, consistent with other white cyclamen cultivars (Takamura et al. 1995). These results suggested that gra50 has defects in the biosynthetic pathway from dihydrokaempferol to anthocyanin. We carried out degenerate PCR and identified four homologues of anthocyanin biosynthesis genes in C. graecum: CgraF3'5'H, CgraDFR1, CgraDFR2 and
CgraANS. The expression of CgraDFR2 was greatly decreased in gra50 petals relative to C. graecum. Several deletions and insertions were found in the upstream sequence of the CgraDFR2 in gra50, and these changes were likely to have caused the reduced expression levels (Akita et al. 2010).

\section{Glucosyltransferase in C. purpurascens}

Glucosylation of anthocyanidins or anthocyanins increases the stability of anthocyanins and modifies color. Another red-purple-flowered mutant, 'KMMv3' (Figure 1J), was obtained from KM by ion beam irradiation (Ishizaka et al. (2012) in this issue). KMMv3 accumulated malvidin 3-glucoside (Mv3G) as a major anthocyanin component, suggesting defective anthocyanin 5-glucosyltransferase (5GT) activity. No $5 G T$ genes had been isolated from cyclamen. We isolated three 5GT homologues from C. purpurascens. Cpur5GT1 and Cpur5GT3 were expressed only in petals, while Cpur5GT2 was expressed in both petals and leaves (Figure 2). Phylogenetic analysis showed that Cpur5GT2 was most similar to other known functional 5GT proteins. We confirmed that Cpur5GT2 had glucosyltransferase activity in vitro using Mv3G as a substrate (Akita et al. unpublished results). The enzymatic activity of Cpur5GT1 and CPur5GT3 remain to be analyzed. Comparative analysis of $\mathrm{KM}$ and KMMv3 would offer us more information about 5GTs in cyclamen.

The UDP-glucose-dependent anthocyanidin 3-glucosyltransferase (3GT) and UDP-glucosedependent 5GT are major enzymes in the glucosylation reaction. Recently, a novel glucosylation reaction by acyl-glucose-dependent glucosyltransferase was reported in carnation and delphinium (Matsuba et al. 2010). Glucosylation reactions at both the 3- and 5- positions are likely to be dependent on UDP-glucose in cyclamen, because a crude cell extract showed glycosylation activity in vitro when UDP-glucose, but not acyl-glucose, was used as a donor (Sasaki et al. personal communication). Recently, Okada et al. (2011) also reported that $3 G T$ homologues were not expressed at detectable levels in white-flowered cyclamen variety 'Pure white'. These data suggest that both 3GT and 5GT are involved in flower color in cyclamen, as well as in other plants.

\section{Glutathione S-transferase in C. purpurascens}

Anthocyanins generally accumulate in acidic vacuoles after their synthesis in the cytosol. A member of the glutathione S-transferase (GST) gene family is known to be involved in vacuolar accumulation of anthocyanins in many plant species (Kitamura 2006). Loss-of-function of the anthocyanin-related GST results in less pigmentation because the uptake of flavonoid molecules into vacuoles is disrupted (Kitamura et al. 2010). To identify 
anthocyanin-related GSTs in cyclamen, four candidate genes were isolated from immature KM petals. Molecular functional analysis demonstrated that one of the four candidates functioned to accumulate anthocyanins in vivo (Kitamura et al. 2012). In carnation, a mutant of an anthocyanin-related GST accumulated fewer anthocyanins in petals, resulting in paler flowers, than the original plant (Sasaki et al. (2012) in this issue). Screening of mutants for the anthocyanin-related GST gene would help to develop new fragrant cyclamen cultivars with paler flowers.

\section{Anthocyanin pathway mutation map in cyclamen}

Figure 1 depicts the cyclamen wild species, cultivars, and mutants used in this research project beside the anthocyanin biosynthesis pathway. The yellow-flowered cyclamen, 'Golden boy' (GB; Figure 1E) and ion257 mutant (Figure $1 \mathrm{~F}$ ), accumulated chalcone 2 '-glucoside (Ch2G) and were thought to have defective CHI activity, as described below. The white-flowered variant gra50 (Figure 1G) did not accumulate anthocyanin, and this phenotype may have had reduced DFR expression. The white-flowered mutant 'KOpw' (Figure 1H) was obtained from fragrant cyclamen 'Kokou-no-kaori' (KO; Figure 1L), although the gene responsible for this mutant was not identified. Either a defective DFR or $3 G T$ could have caused white flowers in KOpw, as was suggested for a white-flowered cultivar (Okada et al. 2011). The red-purple-flowered mutants, KMrp (Figure 1I) and KMMv3 (Figure 1J), accumulated Dp3,5dG and Mv3G, respectively. KMrp expressed no AMT. KMMv3 was thought to have defective 5GT activity. Although the KMrp and KMMv3 accumulated different types of anthocyanins, they could not be distinguished visually from one another. The color of cyclamen petals containing Mv3,5dG was also affected by copigmentation with flavonols (Nakayama et al. (2012) in this issue). These facts suggest that, while flower color variations were mainly due to the types and amounts of anthocyanins produced and to mutations of the pathway genes, modifying anthocyanins also significantly affected flower color.

\section{Effectiveness of PCR screening at early growth stages}

The challenge to mutation breeding in cyclamen is that the plants generally take two years after the irradiation of seeds or cultured tissues to produce flowers that can be evaluated for desirable traits. We assessed the effectiveness of PCR screening of mutant candidates at early growth stages. GB is a yellow-flowered cyclamen cultivar. The CHI gene of GB has been inactivated by an insertion, and GB petals accumulate Ch2G (Miyajima et al. 1991). To develop yellow-flowered fragrant cyclamens, hybrids of $\mathrm{GB} \times C$. purpurascens were
A

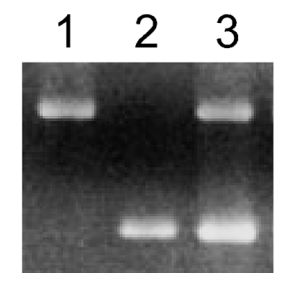

B

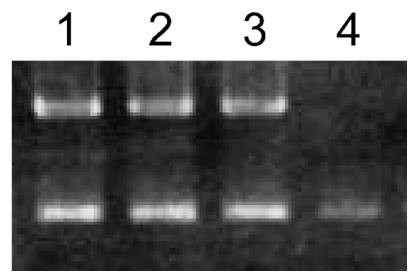

Figure 3. Identification of two different $\mathrm{CHI}$ genes by multiplex genomic PCR. A, PCR products from Cyclamen purpurascens (lane 1), cyclamen cultivar 'Golden boy', GB (lane 2) and a hybrid of $\mathrm{GB} \times C$. purpurascens (lane 3). Upper and lower bands represent PCR products from C. purpurascens and GB, respectively. $\mathrm{CHI}$ gene-specific primers and a primer that was specific for the insertion sequence of the $\mathrm{CHI}$ from GB were used for PCR. B, Detection of a deletion mutant from hybrid $(\mathrm{GB} \times$ C. purpurascens) irradiated with ion beams. Lanes 1 and 2 represent PCR products from a normal leaf and a yellow-green leaf, respectively, taken from a single chimeric mutant. Lanes 3 and 4 represent the same set for another chimeric mutant.

irradiated with ion beams. Petals of the hybrid plants had light pink coloration, because the $\mathrm{CHI}$ gene from $\mathrm{C}$. purpurascens augmented the anthocyanin biosynthesis pathway. We anticipated that a yellow-flowered fragrant cyclamen could be obtained when the $C H I$ gene from $C$. purpurascens was inactivated by ion beam irradiation. During the screening, eight of 1,306 regenerated plants had yellow-green leaves (Ishizaka et al. (2012) in this issue). All eight were chimeric, i.e., part of the plant developed yellow-green leaves while the rest of the same plant developed normal leaves that accumulated anthocyanin on their abaxial sides. Some of the chimeric mutants came into flower, and Ch2G was detected in their yellow petals (Figure 1F; Nakayama et al. personal communication).

We confirmed that multiplex genomic PCR can distinguish the two different $\mathrm{CHI}$ genes in the hybrid cyclamen (Figure 3A). We tested two chimeric mutants that had both normal and yellow-green leaves. In one of the two chimeric mutants, two different $C H I$ genes were detected in both normal and yellow-green leaves (Figure $3 \mathrm{~B}$, lanes 1 and 2). In the other chimeric mutant, $\mathrm{CHI}$ from GB was detected in both normal and yellow-green leaves and $\mathrm{CHI}$ from C. purpurascens was detected only in normal leaves (Figure 3B, lanes 3 and 4). These facts suggest that yellow-green leaves are associated with defective $\mathrm{CHI}$ activity and also that PCR screening could detect some mutants. Although we must assess what fraction of the mutants have the deletion, our results suggest that PCR screening can identify ion beaminduced deletion mutants of a specific gene using about 1,000 mutant plants.

\section{Perspectives}

We believe that the studies described here are a step toward more efficient and controlled mutation breeding 
with ion beams. Stepwise trait improvement takes advantage of ion beams, which can induce mutations with very low irradiation doses. Several studies have demonstrated that ion beams are effective in achieving desired phenotypes in a stepwise manner. Stepwise trait improvement is expected to be especially effective for plants that cannot be cross bred and/or that have highly duplicated genomes. We demonstrated that the direction of mutation could be controlled by pretreatment in petunia. To establish this technique for widespread use, we must verify its effectiveness in other plants and also must elucidate the underlying molecular mechanism. The selection of appropriate starting plant material is also essential to efficient mutation breeding. Okamura et al. (2012, in this issue) succeeded in selecting suitable starting material from hundreds of carnation strains by focusing on the anthocyanin malyl transferase gene. We have identified new cyclamen genes that affect flower coloration. Analyses of cyclamen mutants demonstrated that flower color corresponds to the types and amounts of anthocyanins produced, although modifying anthocyanins also significantly affected flower coloration. Accumulation of knowledge about the relationship between gene mutations and phenotypic traits will help us to anticipate what mutant traits can be obtained from plant material of interest.

\section{Acknowledgements}

We thank all who participated in this research project. Many of the studies described here were supported by a Research and Development Program for New Bio-industry Initiatives grant from the Bio-oriented Technology Research Advancement Institution (BRAIN), Japan.

\section{References}

Akita Y, Ishizaka H, Nakayama M, Shimada A, Kitamura S, Hase Y, Narumi I, Tanaka A (2010) Comparative analysis of floral pigmentation between wild-type and white-flowered varieties of Cyclamen graecum. J Hortic Sci Biotechnol 85: 437-443

Akita Y, Kitamura S, Hase Y, Narumi I, Ishizaka H, Kondo E, Kameari N, Nakayama M, Tanikawa N, Morita Y, Tanaka A (2011) Isolation and characterization of the fragrant cyclamen O-methyltransferase involved in flower coloration. Planta 234: 1127-1136

Hara M, Oki K, Hoshino K, Kuboi T (2003) Enhancement of anthocyanin biosynthesis by sugars in radish (Raphanus sativus) hypocotyl. Plant Sci 164: 259-265

Hase Y, Okamura M, Takeshita D, Narumi I, Tanaka A (2010) Efficient induction of flower-color mutants by ion beam irradiation in petunia seedlings treated with high sucrose concentration. Plant Biotechnol 27: 99-103

Iizuka M, Yoshihara R, Hase Y (2008) Development of commercial variety of Osteospermum by a stepwise mutagenesis by ion beam irradiation. JAEA-Rev 2008-055/JAEA Takasaki Ann Rep 2007: 65

Ishizaka H (2008) Interspecific hybridization by embryo rescue in the genus Cyclamen. Plant Biotechnol 25: 511-519
Ishizaka H, Kondo E, Kameari N (2012) Production of novel flower color mutants from the fragrant cyclamen (Cyclamen persicum $\times$ C. purpurascens) by ion-beam irradiation. Plant Biotechnol 29: 201-208

Joint FAO/IAEA Programme, Mutant Variety Database. http:// mvgs.iaea.org/default.aspx

Kitamura S (2006) Transport of flavonoids. In: Grotewold E (ed) The Science of Flavonoids. Springer, New York, pp 123-146

Kitamura S, Akita Y, Ishizaka H, Narumi I, Tanaka A (2012) Molecular characterization of an anthocyanin-related glutathione $S$-transferase gene in cyclamen. J Plant Physiol 169: 636-642

Kitamura S, Matsuda F, Tohge T, Yonekura-Sakakibara K, Yamazaki M, Saito K, Narumi I (2010) Metabolic profiling and cytological analysis of proanthocyanidins in immature seeds of Arabidopsis thaliana flavonoid accumulation mutants. Plant J 62: 549-559

Kondo E, Nakayama M, Kameari N, Tanikawa N, Morita Y, Akita Y, Hase Y, Tanaka A, Ishizaka H (2009) Red-purple flower due to delphinidin 3,5-diglucoside, a novel pigment for Cyclamen spp., generated by ion-beam irradiation. Plant Biotechnol 26: 565-569

Matsuba Y, Sasaki N, Tera M, Okamura M, Abe Y, Okamoto E, Nakamura H, Funabashi H, Takatsu M, Saito M, Matsuoka H, Nagasawa K, Ozeki Y (2010) A novel glucosylation reaction on anthocyanins catalyzed by acyl-glucose-dependent glucosyltransferase in the petals of carnation and delphinium. Plant Cell 22: 3374-3389

Mita S, Murano N, Akaike M, Nakamura K (1997) Mutants of Arabidopsis thaliana with pleiotropic effects on the expression of the gene for $\beta$-amylase and on the accumulation of anthocyanin that are inducible by sugars. Plant J 11: 841-851

Miyajima I, Maehara T, Kage T, Fujieda K (1991) Identification of the main agent causing yellow color of yellow-flowered cyclamen mutant. J Jpn Soc Hort Sci 60: 409-414

Nagatomi S, Tanaka A, Watanabe H, Tano S (1997) Chrysanthemum mutants regenerated from in vitro explants irradiated with ${ }^{12} \mathrm{C}^{5+}$ ion beam. Institute of Radiation Breeding, Technical News No. 60

Nakayama M, Tanikawa N, Morita Y, Ban Y (2012) Comprehensive analyses of anthocyanin and related compounds to understand flower color change in ion-beam mutants of cyclamen (Cyclamen spp.) and carnation (Dianthus caryophyllus). Plant Biotechnol 29: 215-221

Okada T, Iizuka M, Yoshihara R, Hase Y, Narumi I (2009) Development of commercial variety of Osteospermum by a stepwise mutagenesis by ion beam irradiation. JAEA-Rev 2009041/JAEA Takasaki Ann Rep 2008: 73

Okada S, Narumi T, Fukai S, Takamura T (2011) Main mechanism causing while petal color in white-flowered cyclamen. Hort Res 10 (Suppl 1): 222, in Japanese

Okamura M, Umemoto N, Onishi N (2012) Breeding glittering carnations by an efficient mutagenesis system. Plant Biotechnol 29: 209-214

Sasaki N, Nishizaki Y, Uchida Y, Wakamatsu E, Umemoto N, Momose M, Okamura M, Yoshida H, Yamaguchi M, Nakayama M, Ozeki Y, Itoh Y (2012) Identification of the glutathione $S$-transferase gene responsible for flower color intensity in carnations. Plant Biotechnol 29: 223-227

Sato T, Ohya Y, Hase Y, Tanaka A (2006) Studies on flower color and morphological mutations from chrysanthemum in vitro explants irradiated with ion beams. JAEA-Rev 2005-001/TIARA Ann Rep 2004: 74-75

Shikazono N, Suzuki C, Kitamura S, Watanabe H, Tano S, Tanaka 
A (2005) Analysis of mutations induced by carbon ions in Arabidopsis thaliana. J Exp Bot 56: 587-596

Takamura T, Tomihama T, Miyajima I (1995) Inheritance of yellowflowered characteristic and yellow pigments in diploid cyclamen (Cyclamen persicum Mill.) cultivars. Sci Hortic (Amsterdam) 64: $55-63$

Takamura T, Aizawa M, Kim SY, Nakayama M, Ishizaka H (2000) Inheritance of flower color and pigment in cross between cyanic and acyanic cyclamen. J Jpn Soc Hort Sci 69: 453, in Japanese

Takamura T, Aizawa M, Kim SY, Nakayama M, Ishizaka H (2005) Inheritance of flower pigment in crosses between cyclamen cultivars and Cyclamen purpurascens. Acta Hortic 673: 437-441
Tanaka A, Shikazono N, Hase Y (2010) Studies on biological effects of ion beams on lethality, molecular nature of mutation, mutation rate, and spectrum of mutation phenotype for mutation breeding in higher plants. J Radiat Res (Tokyo) 51: 223-233

Tsukaya H, Ohshima T, Naito S, Chino M, Komeda Y (1991) Sugar-dependent expression of the CHS-A gene for chalcone synthase from petunia in transgenic Arabidopsis. Plant Physiol 97: 1414-1421

Ueno K, Shirao T, Nagayoshi S, Hase Y, Tanaka A (2006) Additional improvement of chrysanthemum using ion beam re-irradiation. JAEA-Rev 2005-001/TIARA Ann Rep 2004: 60-62 